\title{
Etude de la mobilité du calcium dans le lait à l'aide du calcium 45
}

\author{
par \\ A. PIERRE, G. BRULE et J. FAUQUANT
}

\begin{abstract}
R és u m é
L'échangeabilité du calcium dans le lait a été étudiée à l'aide de ${ }^{45} \mathrm{Ca}$.

Une étude cinétique a montré que le calcium lié aux phosphosérines est échangé en moins d'une minute. Le calcium lié au phosphate colloïdal a une échangeabilité différente selon qu'il est lié à la première acidité (échanges en moins d'une minute) ou à la deuxième ( $70 \%$ est échangé en $24 \mathrm{~h})$. Celui lié à la troisième n'est pas échangeable. Un changement de la température ou de l'état physicochimique du lait entraîne une modification de l'échangeabilité. Les relations avec l'état d'ionisation du phosphate sont discutées. Mots clés : ${ }^{45} \mathrm{Ca}$ - Echangeabilité - Lait - Phosphate Ca.
\end{abstract}

\section{S u m m a r y}

STUDY OF Ca EXCHANGEABILITY IN MILK WITH ${ }^{45} \mathrm{Ca}$

${ }^{45} \mathrm{Ca}$ was used to study calcium exchangeability in milk. Time required to exchange all the calcium bound to phosphoserines was less than one minute. Calcium engaged in collö̈dal phosphate was only partly exchangeable. Calcium bound to the first acidity of phosphate was exchanged in less than one minute, the one bound to the second acidity was slowly exchanged $(70 \%$ in $24 \mathrm{~h})$ and the one bound to the third was not exchanged.

Changing temperature of milk or $p H$ alters calcium exchangeability probably in accordance with phosphate ionization. Technological consequences of calcium exchangeability variations are discussed. Key words: ${ }^{45} \mathrm{Ca}$ - Exchangeability - Milk - Ca phosphate.

I.N.R.A., Laboratore de Recherches de Technologie Laitière, 65, rue de SaintBrieuc - 35042 Rennes cedex. 


\section{INTRODUCTION}

Dans le lait, la plus grande partie du calcium (70\%) est associée à la micelle de caséine, soit liée directement aux phosphosérines, soit sous forme de phosphate de calcium colloïdal participant à la structure micellaire (Pyne et Mc Gann, 1960). Le reste du calcium (30\%) se trouve à l'état soluble, essentiellement sous forme de citrate de calcium dans la phase aqueuse (ultrafiltrable).

Dans un lait de composition donnée, la proportion de sels micellaires et de sels solubles est sous la dépendance de la solubilité du phosphate de calcium dans la phase aqueuse, donc conditionnée par l'état physico-chimique du lait. Si les conditions de température et de $\mathrm{pH}$ changent, le rapport des concentrations des sels micellaires et des sels solubles évolue vers un nouvel équilibre.

Dans un lait ayant atteint un tel équilibre des concentrations en phosphate de calcium, il existe une grande mobilité ionique du calcium entre phase soluble et phase micellaire (Maeno, 1956). Cet échange n'altère pas la répartition globale des concentrations. Le calcium lié directement aux caséines (phosphosérines) serait totalement échangeable, par contre celui qui entre dans la constitution du phosphate de calcium colloïdal ne le serait que partiellement. $\mathrm{Au}$ total, environ $65 \%$ du calcium micellaire serait échangeable (Yamauchi et coll., 1969).

Certains traitements appliqués au lait entraînent une modification dans les possibilités d'échange du calcium. Les observations sont très différentes selon que l'on considère l'échangeabilité du calcium pendant l'application du traitement ou après retour aux conditions initiales (effet rémanent). Un traitement thermique à $100^{\circ} \mathrm{C}$ pendant $30 \mathrm{~min}$ ne modifie pas significativement l'échangeabilité du lait, lorsque le test est fait après retour aux conditions initiales (Yamauchi et Yoneda, 1977). Par contre, d'après l'étude de Sindhu et Roy (1982) sur le lait de bufflonne, il apparaît que pendant l'application du traitement, l'échangeabilité du calcium est fortement augmentée.

Le travail que nous avons entrepris était destiné à préciser les données concernant la cinétique des échanges du calcium et à compléter l'étude de l'influence des traitements technologiques sur cette cinétique : traitements thermiques, variation du $\mathrm{pH}$, concentration en protéines, addition de sels minéraux.

\section{MATERIEL ET METHODES}

Le lait utilisé était du lait cru de petit mélange provenant des fermes expérimentales du Ministère de l'Agriculture. Il était écrémé 
sur une écrémeuse centrifuge, puis additionné d'azide de sodium $(0,02 \% \mathrm{p} / \mathrm{v})$ comme agent conservateur. Le lait sans phosphate colloïdal était préparé selon la méthode de Pyne et Mc Gann (1960) : le lait refroidi à $2^{\circ} \mathrm{C}$ était acidifié à $\mathrm{pH} 4,9$ par addition d'acide chlorhydrique $3 \mathrm{~N}$ et dialysé 3 jours à $2^{\circ} \mathrm{C}$ contre le lait initial. Dans certaines expériences, le $\mathrm{pH}$ des laits a été modifié par addition de $\mathrm{NaOH} 2 \mathrm{~N}$ ou de $\mathrm{HCl} 3 \mathrm{~N}$. L'ultrafiltration du lait a été réalisée à $20^{\circ} \mathrm{C}$ sur un module de laboratoire Romicon de $1,5 \mathrm{~m}^{2}$ de surface membranaire, équipé de membranes PM 50; la durée de 1'ultrafiltration était de 1 h 30 environ. La dilution du lait était effectuée par addition d'ultrafiltrat. Les traitements thermiques ont été réalisés par immersion des échantillons dans un bain-marie. Les chauffages aux températures supérieures à $100^{\circ} \mathrm{C}$ étaient effectués à l'autoclave.

La séparation de la phase soluble des laits ou des rétentats d'ultrafiltration était effectuée à température ambiante à l'aide de membranes d'ultrafiltration coniques Amicon CF 25 au cours d'une centrifugation à $1000 \mathrm{~g}$ pendant $15 \mathrm{~min}$. Dans les expériences de cinétique, pour les prélèvements des 15 premières minutes, les phases solubles étaient obtenues par filtration des laits à l'aide d'une seringue Millipore équipée d'une membrane filtrante Millex GS de porosité $0,22 \mu$. Nous avons vérifié que les ultrafiltrats obtenus selon ce procédé avaient la même composition que ceux obtenus sur cônes. Le dosage du calcium était fait par absorption atomique selon la méthode de Brulé et coll. (1974) et le dosage des protéines par Kjeldahl. Le pH était mesuré à l'aide d'un appareil Radiometer PHM 84.

Le calcium marqué était ajouté sous forme d'une solution de ${ }^{45} \mathrm{CaCl}_{2}$, de manière à obtenir une activité de $3 \mu \mathrm{Ci}$ par litre de lait (correspondant à une addition de moins de $1 \mathrm{mg}$ de calcium par litre de lait). Dans les cas où l'addition de ${ }^{45} \mathrm{Ca}$ était faite avant I'application du traitement au lait, un intervalle de temps d'une heure était ménagé entre l'addition de ${ }^{45} \mathrm{Ca}$ et le début du traitement.

Dans tous les cas (sauf les cinétiques) les échantillons étaient laissés $24 \mathrm{~h}$ à $20^{\circ} \mathrm{C}$ en présence du ${ }^{45} \mathrm{Ca}$ avant la séparation de la phase soluble.

La détermination de la radioactivité était faite à l'aide d'un compteur à scintillation liquide (Beckman LS 3800, mélange scintillant HP). La durée de comptage a été choisie de manière à obtenir un coefficient de variation de $2 \%$ sur les déterminations. Le comptage était effectué sur $1 \mathrm{ml}$ de lait ou d'ultrafiltrat, sauf dans le cas des cinétiques où le volume d'ultrafiltrat était de l'ordre de $0,2 \mathrm{ml}$, la quantité exacte étant déterminée par pesée.

Les résultats ont été exprimés soit directement par le rapport de l'activité soluble à l'activité totale (en pourcentage), soit en calculant la proportion de calcium échangeable dans le cacium colloïdal total, selon la formule utilisée par Yamauchi et coll. (1969) : 


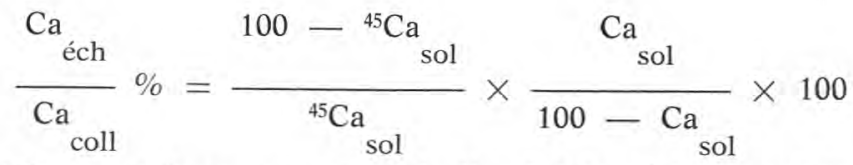

où ${ }^{45} \mathrm{Ca}_{\mathrm{s} 01}$ est le pourcentage de l'activité totale du calcium 45 restant dans la phase soluble, $\mathrm{Ca}_{\mathrm{s} \text { o1 }}$ est le pourcentage du calcium dans la phase soluble par rapport au calcium total du lait.

Nous avons également exprimé le calcium échangeable par rapport au calcium total du lait



La teneur en calcium total lié aux sérines dans le lait normal et les laits dilués (tab. 1) a été déterminée à partir de l'analyse du lait sans phosphate colloïdal, en faisant la différence entre le calcium total et le calcium soluble de ce lait. Pour les laits dilués, il a été tenu compte du facteur de dilution.

\section{RESULTATS}

\section{Cinétique des échanges}

Pour étudier la cinétique des échanges, du calcium marqué était ajouté à un lait, soit normal, soit préalablement dilué par de l'ultrafiltrat, puis la radioactivité résiduelle de la fraction ultrafiltrable était suivie au cours du temps. Au moment de l'addition du calcium marqué, toute la radioactivité $(100 \%)$ se trouve dans la phase soluble. Lorsque des échanges s'effectuent avec la phase micellaire, la radioactivité de l'ultrafiltrat diminue.

Dans une première expérience, les échanges se produisant pendant les $10 \mathrm{~min}$ suivant l'addition du ${ }^{45} \mathrm{Ca}$ ont été étudiés sur des laits dilués au $1 / 2$ et au 1/5. Le lait normal n'a pas été étudié, car la technique de filtration rapide utilisée ne permettait pas de séparer une phase soluble à partir du lait non dilué. Nous avons réalisé le premier prélèvement 1 min environ après l'addition de la radioactivité, puis des prélèvements successifs à des intervalles de 1 à 2 min. Le pourcentage de l'activité se trouvant dans la phase soluble est identique pour tous les échantillons analysés. Il est de 83,7\% $\%$ 2,7 $(n=7)$ pour le lait dilué au $1 / 5$ et de $63,3 \% \pm 4(n=5)$ pour le lait dilué au 1/2. Au temps zéro, lors de l'addition de la radioactivité 
(sous forme de ${ }^{45} \mathrm{CaCl}_{2}$ ), $100 \%$ de l'activité se trouve dans la phase soluble du lait. En $1 \mathrm{~min}$ (ou moins) elle diminue respectivement de $16,3 \%$ et de $36,7 \%$ pour les laits dilués $1 / 5$ et au $1 / 2$; dans les 9 min suivantes, on ne note pas de variation mesurable.

Une deuxième expérience a permis de suivre l'évolution de l'activité de la phase soluble d'un lait normal ou dilué (au $1 / 2$ et $1 / 5$ ) pendant $24 \mathrm{~h}$ (fig. 1). Le premier prélèvement a été fait $15 \mathrm{~min}$ après l'addition du ${ }^{45} \mathrm{Ca}$. L'activité de la phase soluble diminue jusqu'à un palier égal à $74 \%, 56 \%$ et $40 \%$ respectivement, pour le lait dilué au $1 / 5$, au $1 / 2$ et non dilué.

Sur la figure 1, sont également reportés des résultats d'une cinétique d'échange du calcium dans du lait sans phosphate colloïdal (lait S.P.). Dans le tableau 1, nous avons regroupé les données concernant la composition en calcium des échantillons de lait normal, lait dilué et lait S.P. utilisés pour la cinétique (fig. 1) et reporté les calculs de la répartition du calcium échangé entre les différents constituants du lait.

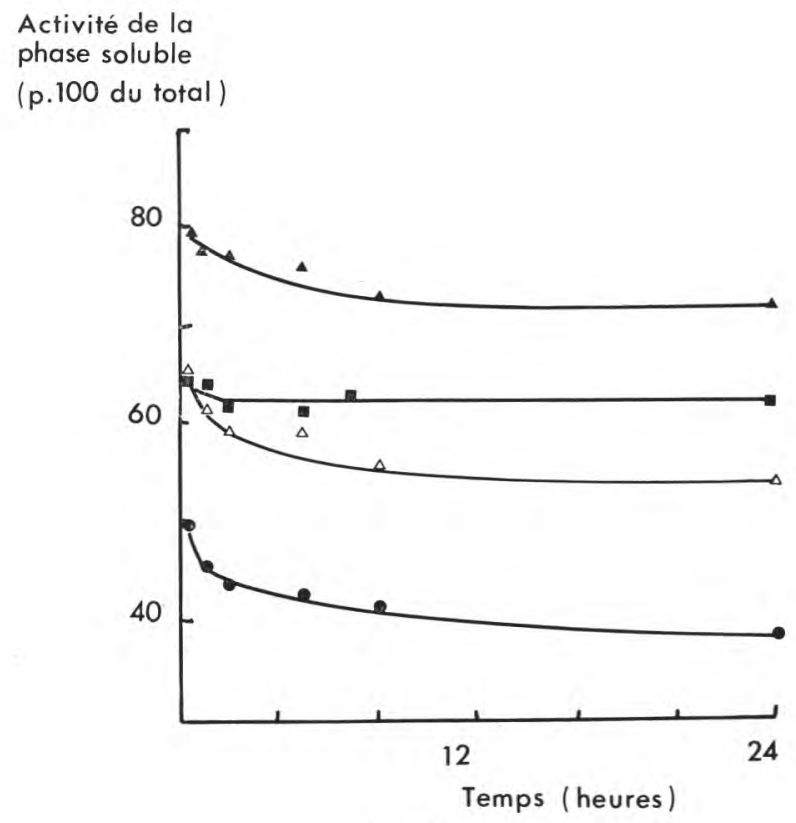

fig. 1

Evolution au cours du temps de la radioactivité de la phase soluble d'un lait (en $\%$ de la radioactivité totale ajoutée). ( $\bullet$ lait non dilué ; $(\Delta)$ lait dilué au

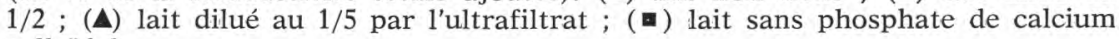
colloïdal. 


\section{TABLEAU 1}

Répartition du calcium échangeable $(\mathrm{mM} / \mathrm{kg})$ dans du lait sans phosphate inorganique colloïdal (lait S.P.), du lait dilué avec de l'ultrafiltrat et du lait normal

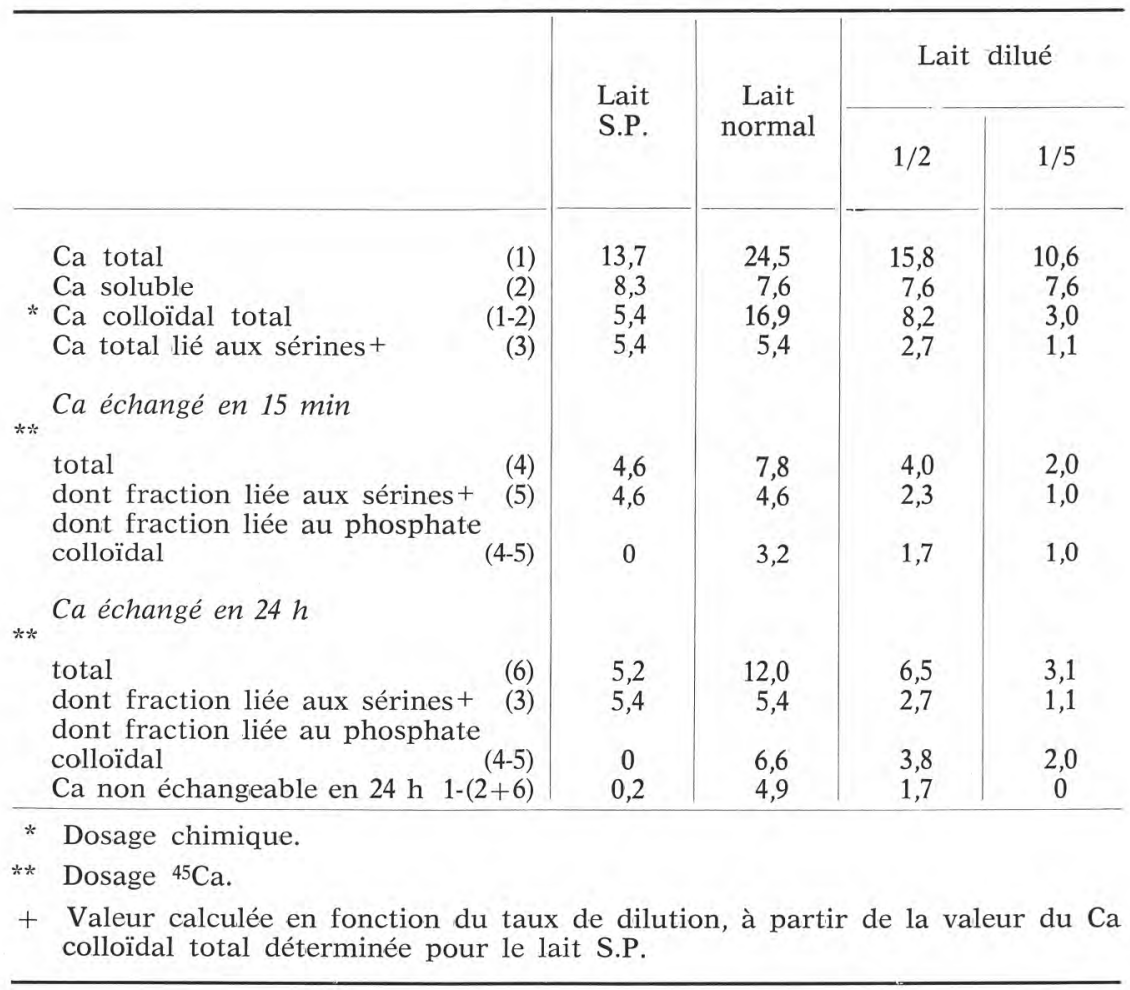

\section{TABLEAU 2}

Taux d'échangeabilité du calcium micellaire dans du lait ayant subi un traitement thermique (en \% du calcium micellaire total)

\begin{tabular}{l|c|c}
\hline \multicolumn{1}{c|}{ Traitement du lait } & $\begin{array}{c}45 \mathrm{Ca} \text { ajouté avant } \\
\text { traitement thermique }\end{array}$ & $\begin{array}{c}45 \mathrm{Ca} \text { ajouté après } \\
\text { traitement thermique }\end{array}$ \\
\hline $20^{\circ} \mathrm{C}$ (témoin) & 71,7 & 72,8 \\
$72^{\circ} \mathrm{C}-15 \mathrm{~s}$ & 72,5 & 73,4 \\
$120^{\circ} \mathrm{C}-20 \mathrm{~min}$ & 100,0 & 49,2 \\
$2^{\circ} \mathrm{C}-3 \mathrm{j}$ & 73,5 & 65,6 \\
\hline
\end{tabular}

Moyenne de trois laits de mélange. 


\section{Influence des traitements thermiques}

Le tableau 2 montre la variation du taux de calcium micellaire échangeable dans des laits soumis à divers traitements thermiques. Lorsque le ${ }^{45} \mathrm{Ca}$ est ajouté avant le chauffage, on obtient une échangeabilité de $100 \%$ au cours du chauffage à $120^{\circ}$; les autres traitements conduisent à des différences non significatives. Lorsque le ${ }^{45} \mathrm{Ca}$ est ajouté après le traitement thermique, on peut mettre en évidence que l'effet rémanent du traitement est faible, sauf dans le cas du lait stérilisé où la teneur en calcium échangeable diminue.

La variation de l'échangeabilité du calcium micellaire a également été étudiée en fonction de la température de chauffage dans l'intervalle $20^{\circ} \mathrm{C}$ à $120^{\circ} \mathrm{C}$ pendant des durées de $30 \mathrm{~min}$. Cette durée ne tient pas compte du temps de montée et de descente de la température. La durée réelle du chauffage est donc plus longue de $30 \%$ environ pour les deux techniques de chauffage utilisées (bain-marie, autoclave). la figure 2 montre que l'échangeabilité du calcium colloïdal augmente avec la température du chauffage. D'un lait à l'autre, la

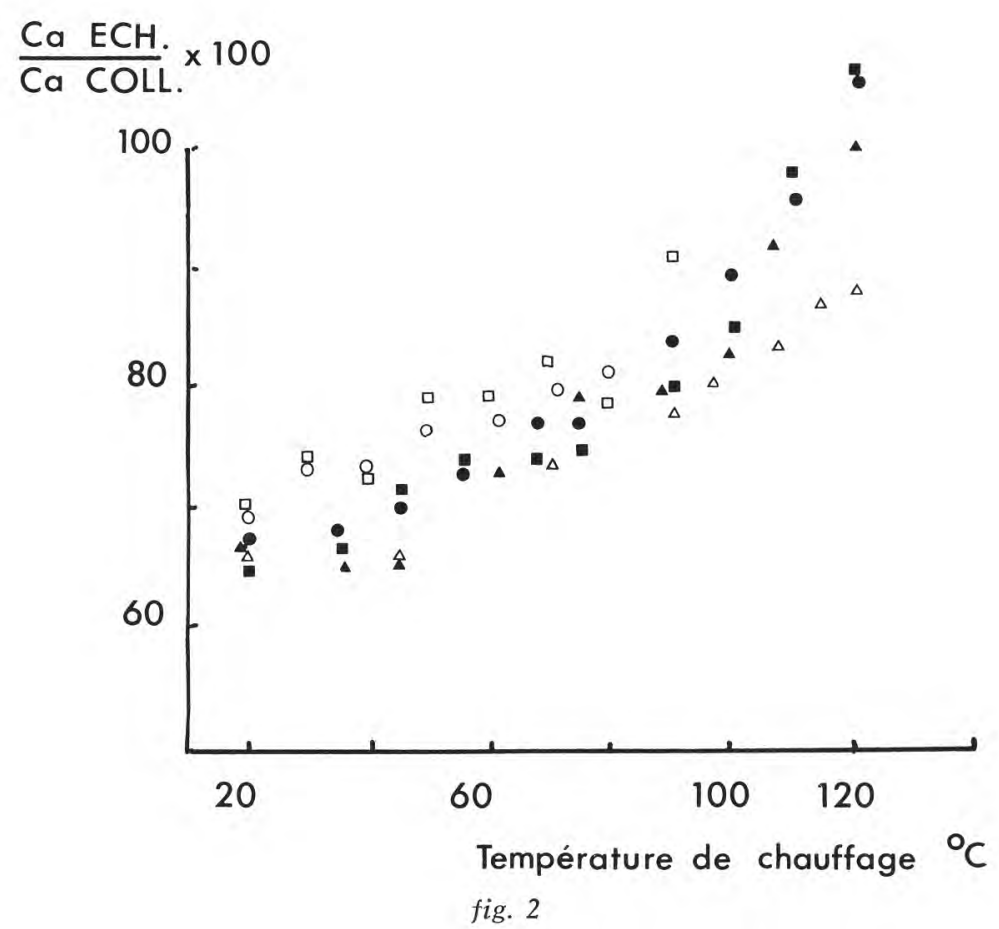

Echangeabilité du calcium colloïdal en fonction de la température pour six laits de mélange. 
teneur en calcium échangeable initiale, déterminée à $20^{\circ} \mathrm{C}$, est variable, mais l'évolution en fonction de la température est similaire pour tous les laits. L'augmentation semble linéaire pour des chauffages à des températures entre $20^{\circ} \mathrm{C}$ et $90^{\circ} \mathrm{C}$. Elle est de $0,22 \%$ par degré $(\mathrm{y}=0,0022 \mathrm{x}+0,62 ; \mathrm{r}=0,85 \mathrm{n}=44)$. Aux températures supérieures, l'augmentation de l'échangeabilité est plus rapide : elle est de $0,6 \%$ par degré $(\mathrm{y}=0,0060 \mathrm{x}+0,29 ; \mathrm{r}=0,90 \mathrm{n}=10 ;$ il n'a pas été tenu compte, pour le calcul, du lait dont le comportement est très différent des autres à température élevée). Nous avons comparé les taux de calcium échangé après deux durées d'incubation, $30 \mathrm{~min}$ et $60 \mathrm{~min}$, à différentes températures. La figure 3 présente les résul-

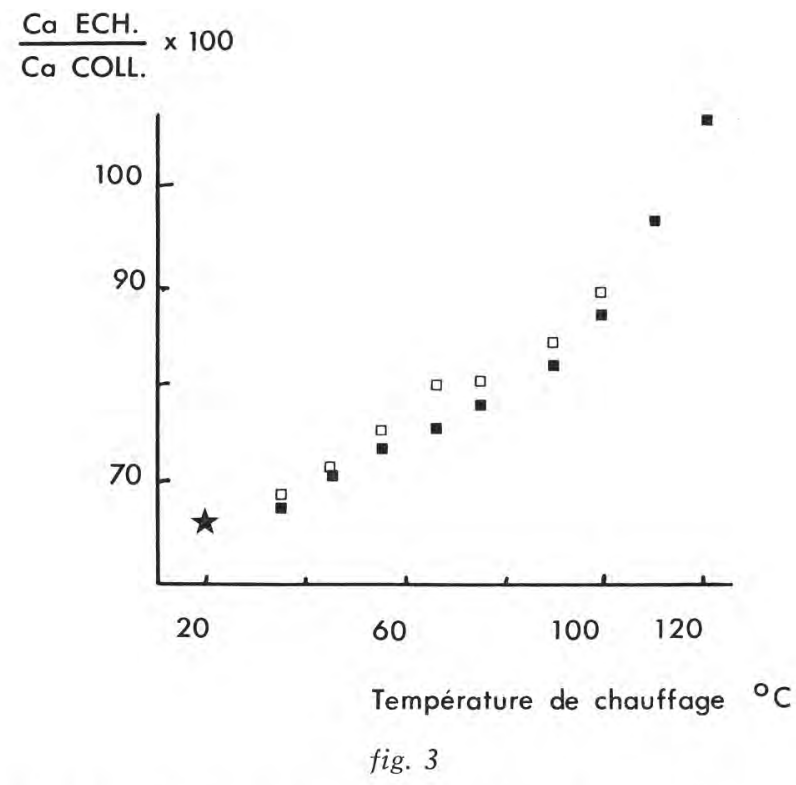

Influence de la durée du chauffage, $20 \mathrm{~min}$. (四) ou $60 \mathrm{~min}$ (ㅁ) sur l'échangeabilité. Moyenne de deux laits.

tats. Chaque point est la moyenne des valeurs obtenues pour 2 laits différents. Le taux de calcium échangé est plus élevé après $60 \mathrm{~min}$ de chauffage qu'après $30 \mathrm{~min}$. La différence entre les deux traitements est en moyenne de $3 \%$ du calcium colloïdal total. Elle est significative au seuil 0,05 .

Par ailleurs, nous avons effectué, à partir des résultats précédents, une représentation du logarithme népérien du rapport $\mathrm{Ca}$ éch/Ca coll en fonction de $\mathrm{l} / \mathrm{T}$ afin de déterminer la pente de la 


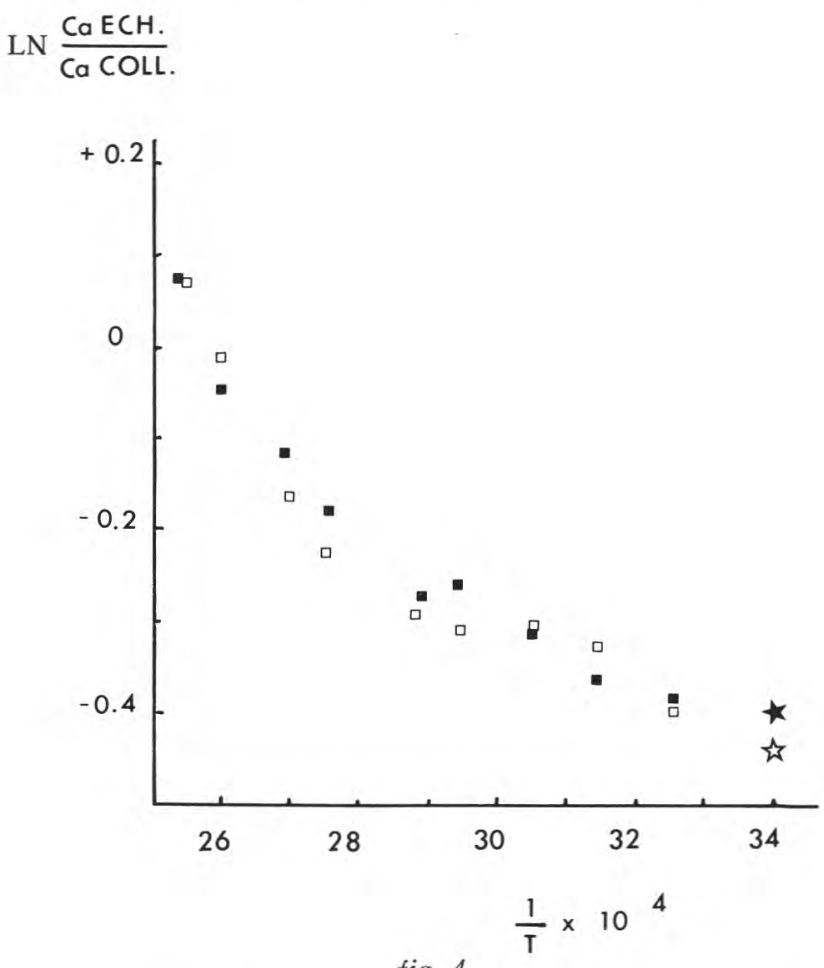

fig. 4

Représentation du logarithme de l'échangeabilité du calcium colloïdal en fonction de $1 /$ T pour deux laits de mélange.

droite obtenue et de calculer l'énergie de la liaison calcium-phosphate en utilisant la loi d'Arrhénius. La figure 4 présente les résultats concernant les deux laits après $30 \mathrm{~min}$ de chauffage. Les points expérimentaux se répartissent selon deux demi-droites avec une inflexion approximativement pour la valeur 0,00275 de $1 / \mathrm{T}$, soit pour la température de $90^{\circ} \mathrm{C}$. Dans l'intervalle de $1 / \mathrm{T}$ de 0,00275 à 0,0034 , nous avons calculé une équation de régression de $-317 \mathrm{l} / \mathrm{T}+0,653(\mathrm{n}=14$, $\mathrm{r}=-0,95$ ) conduisant à une valeur de l'énergie de liaison de - 2,7 KJ/mole de calcium. Le traitement de l'ensemble des données présentées sur la figure 2, concernant six laits, donne une équation de $-3101 / T+0,65(n=44, r=0,85)$, conduisant à une énergie de $-2,6 \mathrm{KJ} / \mathrm{mole}$.

Dans ce cas, le coefficient de corrélation obtenu est plus faible, car il existe une variabilité dans la proportion initiale du calcium échangeable mesurée à $20^{\circ} \mathrm{C}$ qui crée une dispersion des points ; mais l'évolution en fonction de la température est identique. 
Pour l'intervalle de $1 / \mathrm{T}$ de 0,0025 à 0,00275 les points représentés sur la figure 4 correspondent à l'équation $-12631 / \mathrm{T} 3,26$ ( $\mathrm{n}=8$; $\mathrm{r}=-0,98)$ conduisant à une énergie de $-10,6 \mathrm{KJ}$ par mole de calcium.

\section{Influence du pH}

L'influence du $\mathrm{pH}$ du lait sur l'échangeabilité du calcium a été étudiée sur des échantillons de lait dont le $\mathrm{pH}$ avait été ajusté à des valeurs de 7,5 à 4,7 , puis équilibrés pendant $3 \mathrm{~h}$ à $20^{\circ} \mathrm{C}$. Le calcium radioactif était alors additionné et après $24 \mathrm{~h}$ de contact à $20^{\circ} \mathrm{C}$, les phases solubles étaient extraites et leur activité déterminée. Par ailleurs, une série d'échantillons aux mêmes $\mathrm{pH}$ mais non additionnés de ${ }^{45} \mathrm{Ca}$ étaient traités de façon identique afin de déterminer la répartition du calcium dans les laits. Les dosages chimiques permettaient de déterminer le calcium soluble, le calcium

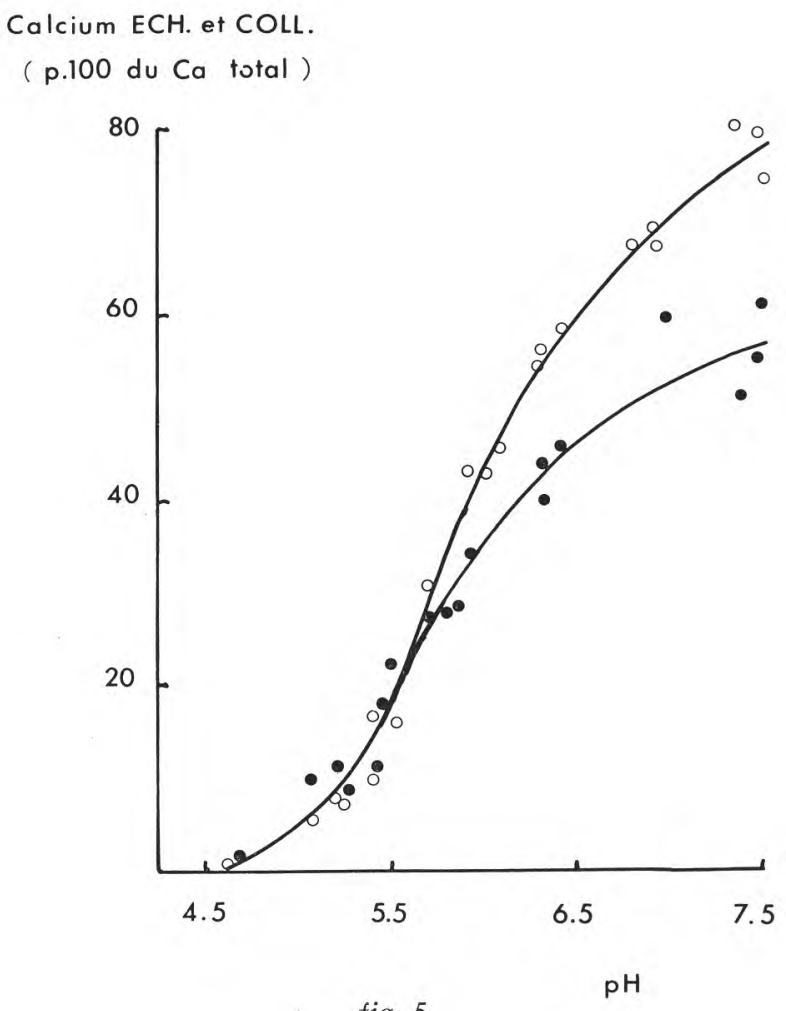

fig. 5

Influence du $\mathrm{pH}$ du lait sur la quantité ( $\mathrm{g} \mathrm{p} . \mathrm{kg}$ ) de calcium échangeable (•) et de calcium colloïdal (O). 
total et, par différence, le calcium colloïdal des laits; à partir des activités, mesurées dans les laits et ultrafiltrats, la teneur en calcium colloïdal échangeable était calculée. Sur la figure 5 sont reportés ces deux ensembles de valeurs pour tous les pH étudiés. On observe que la teneur en calcium colloïdal échangeable du lait diminue avec le $\mathrm{pH}$, comme la teneur en calcium colloïdal. Il est possible à partir des deux courbes de la figure 5 de calculer le rapport du calcium échangeable au calcium colloïdal à chaque $\mathrm{pH}$. On obtient la courbe de la figure 6 qui montre que la proportion de calcium échangeable dans le calcium colloïdal augmente quand le $\mathrm{pH}$ diminue. Tout le calcium est échangeable $(100 \%)$ à $\mathrm{pH} 5,5$ environ. Sur la figure 6 sont également reportés les points correspondant au calcium échangeable calculé selon la formule de Yamauchi et coll. (1969), pour chaque détermination expérimentale. On remarque que leur disper-

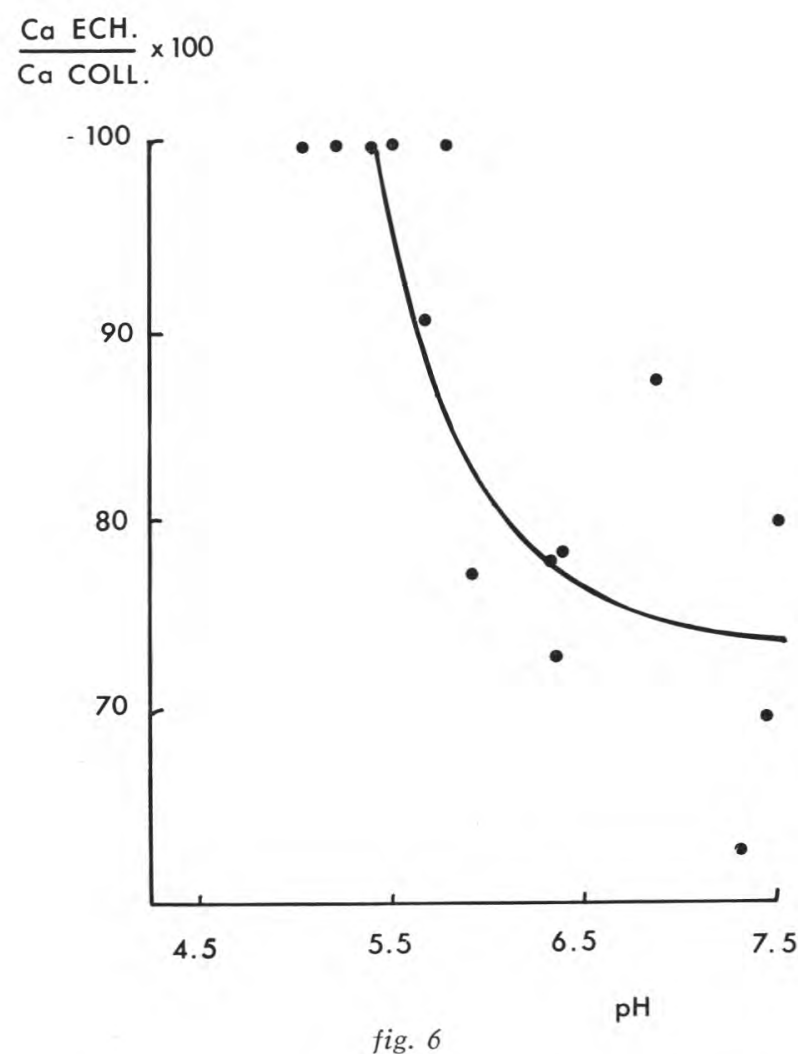

Echangeabilité du calcium colloïdal en fonction du pH du lait. (•), points expérimentaux ; la courbe représente le rapport $\mathrm{Ca}$ éch/Ca coll calculé à partir des deux courbes de la figure 5. 
sion est très importante. En effet, le calcul fait intervenir plusieurs valeurs expérimentales, d'où une erreur de $15 \%$ sur chaque résultat.

\section{Influence de la teneur en protéines}

L'échangeabilité du calcium a été déterminée dans des laits enrichis en protéines par ultrafiltration jusqu'à des teneurs de $53,5 \mathrm{~g} / \mathrm{kg}$ et dans un lait dilué par de l'ultrafiltrat $(17,2 \mathrm{~g} / \mathrm{kg}$ de protéines). La figure 7 montre que le taux de calcium échangeable dans le calcium colloïdal n'est pas modifié par la concentration en protéines (fig. 7a). Par contre, le calcium échangeable représente une proportion de plus en plus importante du calcium total lorsque la teneur en protéines augmente de 20 à $40 \mathrm{~g} / \mathrm{kg}$. Au-delà, la variation est plus faible (fig. 7b).

\section{Influence d'une addition de sels}

Lors de l'addition de chlorure de calcium au lait, on observe une fixation partielle du calcium sur la micelle (Brulé et Fauquant,

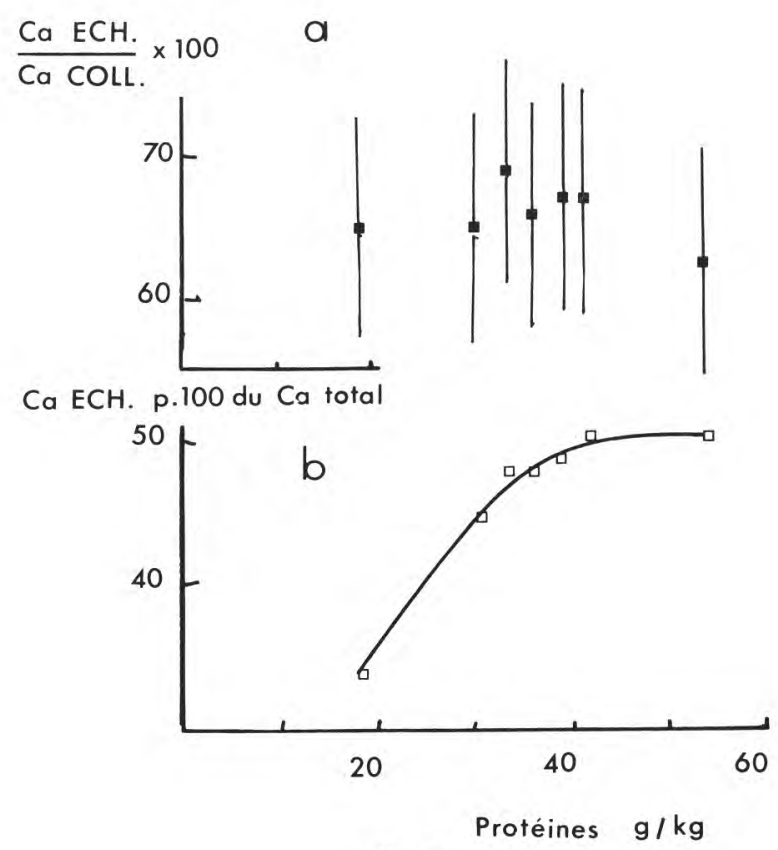

fig. 7

Influence de la teneur en protéines du lait sur l'échangeabilité du calcium colloïdal (a) et sur la proportion de calcium échangeable dans le calcium total du lait (b). 
1981) et une baisse du pH (fig. 8). La teneur en calcium échangeable du calcium colloïdal est peu modifiée par le traitement (fig. 8), on observe cependant une augmentation qui est seulement en partie explicable par l'abaissement du $\mathrm{pH}$.
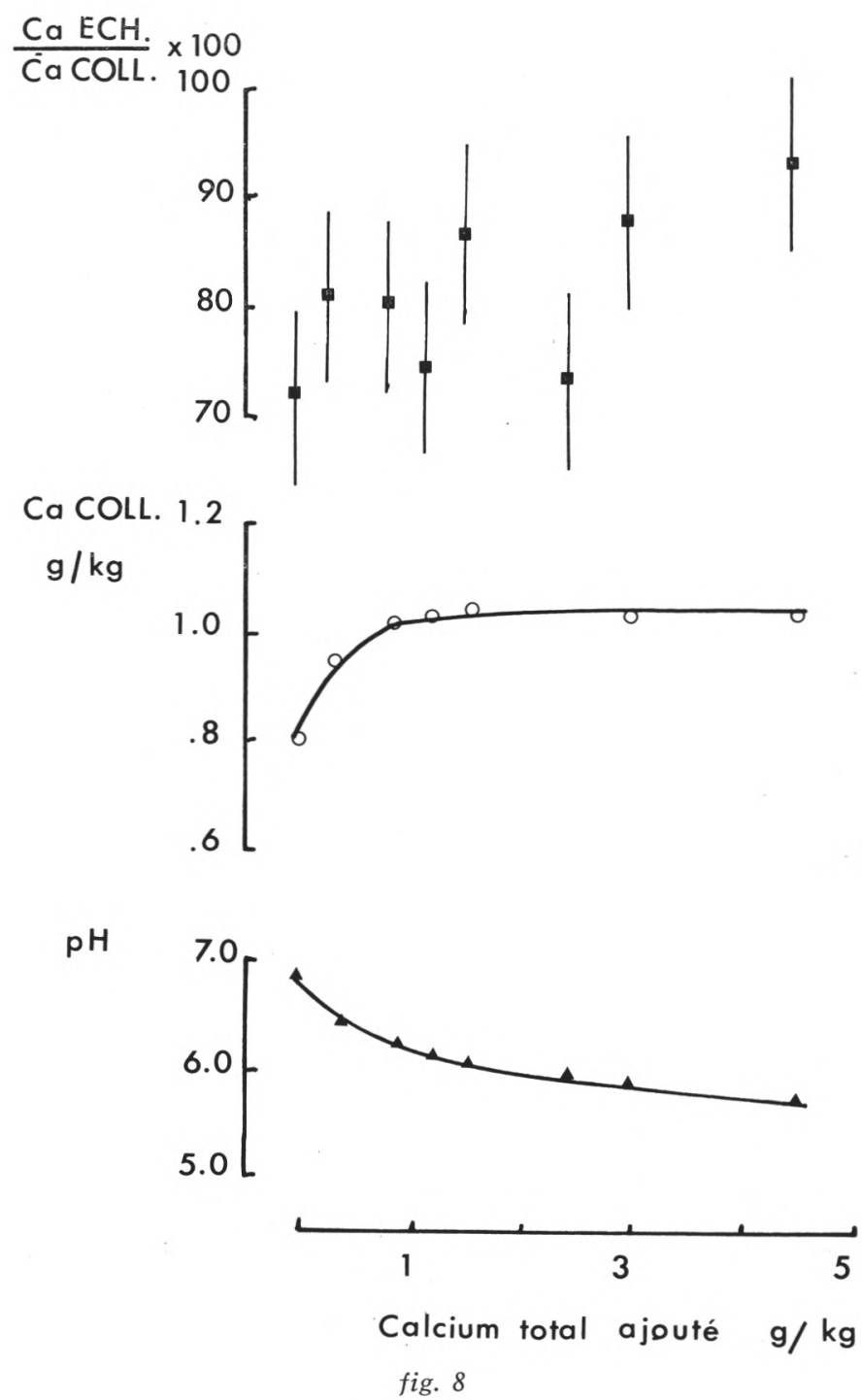

Influence d'une addition de chlorure de calcium sur l'échangeabilité du calcium colloïdal. 
Nous avons également étudié l'incidence d'une addition d'ions citrate à du lait sur l'échangeabilité du calcium colloïdal micellaire. Le citrate était ajouté sous forme d'une solution de citrate de sodium $(0,3$ à $0,5 \mathrm{~g} / \mathrm{ml}$ d'ion citrate, selon l'expérience) dont le $\mathrm{pH}$ était ajusté à 6,80 , c'est-à-dire à un $\mathrm{pH}$ très proche de celui du lait. Des quantités croissantes de la solution de citrate ont été ajoutées à des parties aliquotes du même lait. Tous les échantillons ont ensuite été ajustés au même volume final par addition d'eau. La dilution était de 1,06. Le pH des échantillons de lait était ramené à la valeur de 6,80 après addition du citrate lorsque cela était nécessaire. Le citrate entraînait la solubilisation du calcium et du phosphore micellaire. La teneur résiduelle en calcium micellaire diminue ainsi que la quantité de calcium échangeable (fig. 9). Le rapport du calcium échangeable au calcium colloïdal était dans le lait de $64,5 \% \pm 4$. Pour des teneurs en citrate comprises entre 0 et $15 \mathrm{mM}$ (7 déterminations) il est constant et égal à $63,5 \% \pm 1,5$. Pour des concentrations en citrate supérieures, il diminue (56\% pour $25 \mathrm{mM}$ ).

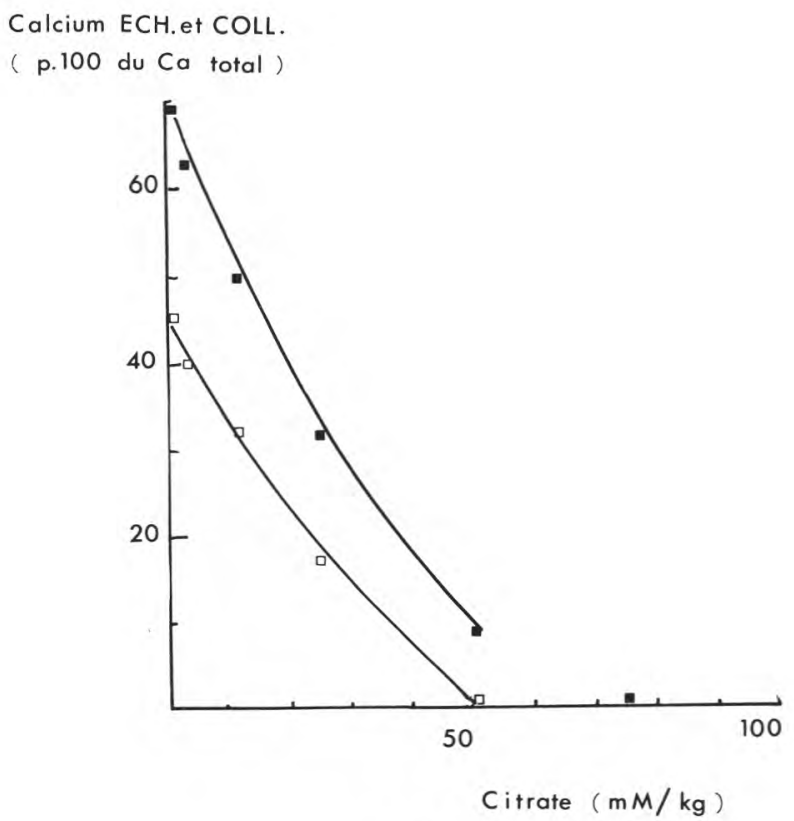

fig. 9

Influence d'une addition de citrate sur l'échangeabilité du calcium colloïdal : (口) Ca colloïdal échangeable ; ( ) Ca colloïdal total. 


\section{DISCUSSION}

Nos résultats ont permis de confirmer que seule une partie du calcium du lait est échangeable et qu'il existe des échanges rapides et des échanges lents (Yamauchi et coll., 1969). Les échanges rapides sont effectués en moins de $1 \mathrm{~min}$, puisque entre 1 et $10 \mathrm{~min}$, nous observons déjà un pallier dans la concentration en ${ }^{45} \mathrm{Ca}$ soluble. A partir de l'exploitation de nos résultats expérimentaux réalisés dans le tableau 1, il apparaît que $7,8 \mathrm{mM}$ de calcium sont échangés dans le lait en moins de $15 \mathrm{~min}$, ce qui correspond à la quasi-totalité du calcium liée aux phosphosérines $(4,6 \mathrm{mM}$ sur un total de 5,4 mM) et à une partie du calcium liée au phosphate colloïdal $(3,2 \mathrm{mM}$ sur un total de $11,5 \mathrm{mM}$ ). Pendant les $24 \mathrm{~h}$ suivantes, la quantité de calcium échangé augmente et après $24 \mathrm{~h}$ elle est de $12 \mathrm{mM}$ au total, dont 6,6 mM de calcium lié au phosphate colloïdal. C'est le déroulement de cet échange que l'on observe sur la figure 1. Une partie du calcium lié au phosphate colloïdal (43\%) n'est donc pas échangeable en $24 \mathrm{~h}$ à $20^{\circ} \mathrm{C}$. Lorsqu'on élève la température du lait, on observe une augmentation de la quantité de calcium échangée rapidement (mesurée après $30 \mathrm{~min}$ de traitement thermique), sans doute en raison de l'augmentation de l'ionisation du phosphate de calcium en fonction de la température. Dans l'intervalle de 20 à $90^{\circ} \mathrm{C}$ la quantité de calcium échangée (de $66 \%$ à $78 \%$ du calcium colloïdal total) correspond à $2 \mathrm{mM}$ de calcium, pour lesquels nous avons déterminé une énergie de liaison de $-2,7 \mathrm{KJ} /$ mole. Entre 90 et $120^{\circ} \mathrm{C}$, la quantité de calcium échangée passe de 80 à $100 \%$ du calcium colloïdal total, soit $3,4 \mathrm{mM}$ de calcium dont l'énergie de liaison a été évaluée à $-10,6 \mathrm{KJ} /$ mole.

On peut penser que les trois liaisons du phosphate de calcium aient des caractéristiques différentes : la liaison correspondant à la première acidité serait échangée très rapidement, en moins de $1 \mathrm{~min}$ à $20^{\circ} \mathrm{C}$. Celle correspondant à la deuxième acidité serait échangée plus lentement : en $24 \mathrm{~h}$ à $20^{\circ} \mathrm{C}$ elle serait seulement partiellement échangée. Par contre, sous l'action d'un chauffage à des températures inférieures à $90^{\circ} \mathrm{C}$, elle serait totalement échangée, l'énergie de liaison étant de $-2,7 \mathrm{KJ} /$ mole. Enfin celle de la troisième acidité serait échangée seulement aux températures de chauffage plus élevées en raison d'une énergie de liaison plus grande, $-10,6 \mathrm{KJ} / \mathrm{mole}$.

Ces observations montrent que sous l'action d'un chauffage suffisamment intense, toutes les liaisons du phosphate de calcium deviennent labiles. Des réarrangements structuraux peuvent alors se produire dans la micelle entre caséines et phosphate colloïdal, qui ne sont sans doute pas réversibles lors du refroidissement. Par ailleurs, le chauffage favorise la transformation du phosphate de calcium micellaire colloïdal en hydroxyapatite (forme cristallisée). Ces transformations permettent d'expliquer le changement dans les 
propriétés technologiques des laits après traitement thermique, en particulier les répercussions sur la coagulation par la présure : allongement du temps de coagulation, diminution de la fermeté du caillé. En effet, le rôle du phosphate de calcium colloïdal natif du lait dans le développement de la fermeté du caillé présure a été mis en évidence depuis longtemps (Pyne et Mc Gann, 1962).

L'ultrafiltration ou l'addition de sels ne modifie pas l'échangeabilité du calcium colloïdal du lait, par contre un changement dans le $\mathrm{pH}$ a une grande incidence. L'acidification provoque une régression de la dissociation des fonctions acides du phosphate colloïdal qui entraîne sa solubilisation partielle. Quand le $\mathrm{pH}$ diminue, l'échangeabilité du calcium micellaire augmente, en partie parce que le rapport calcium lié aux sérines/calcium du phosphate tricalcique augmente, en raison aussi de la régression d'ionisation du phosphate tricalcique. A pH 5,5, tout le calcium restant sur la micelle est échangeable. A ce $\mathrm{pH}$ toutes les liaisons entre le phosphate et le calcium dans la micelle deviennent donc labiles. Le phosphate de calcium colloïdal perd son organisation et sa rigidité et, par là même, sa fonction dans le maintien de la structure micellaire. Une réorganisation spatiale des caséines, libérées des liaisons minérales, pourrait alors se produire avec la formation de sous-micelles, sous l'impulsion dominante des interactions hydrophobes. Les résultats de Goudedranche et coll. (1980) concernant l'évolution de la viscosité des laits ultrafiltrés en fonction du $\mathrm{pH}$ confirment des changements importants de la structure à ce $\mathrm{pH}$.

\section{Remerciements}

Nous remercions J. L. Maubois et J. Hermier pour la lecture critique du manuscrit.

\section{Bibliographie}

Brulé (G.) and Fauquant (J.) (1981). - Mineral balance in skimmilk and milk retentate: effect of physico-chemical characteristics of the aqueous phase, Journal of Dairy Research, 48, 91-97.

Brulé (G.) Maubois (J. L.) et Fauquant (J.) (1974). - Etude de la teneur en éléments minéraux des produits obtenus lors de l'ultrafiltration du lait sur membrane. Le Lait, 54, 600-615.

Goudedranche (H.), Maubois (J. L.), Ducruet (P.) and Mahaut (M.) (1980). Utilization of the new mineral UF membranes for making semi-hard cheeses. Desalination, 35, 243-258.

Maeno (M.) (1956). - Studies on the radioactive substances of milk and milk products, and the combination of $\mathrm{P}$ and $\mathrm{Ca}$ to milk protein using ${ }^{45} \mathrm{Ca}$ and 32P. XIV International Dairy Congress, 1, 130-138.

PYNE (G. T.) and Mc GANN (T. C.A.) (1960). - The colloidal phosphate of milk. II. Influence of citrate. Journal of Dairy Research, 27, 9. 
Pyne (G. T.) and Mc Gann (T.C. A.) (1962). - The influence of the colloidal phosphate of milk on the rennet coagulation. XVI International Dairy Congress, B IV, 1, 611-616.

SindHU (J. S.) and RoY (N. K.) (1982). - Distribution of $45 \mathrm{Ca}$ between soluble and colloidal phases of buffaloes milk as affected by processing. Journal of the Science of Food and Agriculture, 32, 299-302.

Yamauchi (K.), Yoneda (Y.), Koga (Y.) and Tsugo (T.) (1969). - Exchangeability of colloidal calcium in milk with soluble calcium. Agricultural and Biological Chemistry, 33 (6), 907-914.

Yamauchi (K.) and Yoneda (Y) (1977). - Effect of some treatments of milk on the exchangeability of colloidal calcium in milk with soluble calcium. Agricultural and Biological Chemistry, 41 (12), 2395-2399. 